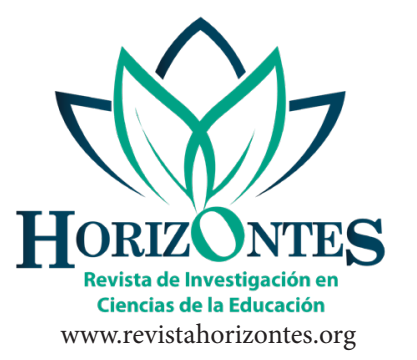

Horizontes. Revista de Investigación en Ciencias de la Educación Https://doi.org/10.33996/revistahorizontes.v6i22.319 Enero-marzo 2022 Volumen 6 / No. 22

ISSN: 2616-7964 ISSN-L: 2616-7964 pp. $108-118$

www.revistahorizontes.org

\title{
El aporte de la pragmática y el sentido del contexto en la comprensión de textos
}

\author{
The contribution of pragmatics and the sense of context in text comprehension \\ O contributo da pragmática e o sentido de contexto na compreensão dos textos
}

ARTíCULO DE INVESTIGACIÓN

Maximina Angélica Deza Sánchez

madezasanchez@gmail.com

https://orcid.org/0000-0003-1558-5139

Universidad César Vallejo, Lima-Perú

Carmen Madaleyne Huamán Ocaña

huamanocañacarmenmadaleyne@gmail.com https://orcid.org/0000-0002-0383-2502

Universidad César Vallejo, Lima-Perú

\section{Jaqueline Jéssica Manzanares Gómez jaquelinejessicamanzanares@gmail.com https://orcid.org/0000-0002-4141-3026 \\ Universidad César Vallejo, Lima-Perú}

Fredy Esmit Guerrero Huamán

huamanocanafredyesmit@gmail.com https://orcid.org/0000-0002-8533-1815

Universidad César Vallejo, Lima-Perú

Artículo recibido 25 de octubre 2021, arbitrado y aceptado 11 de noviembre 2021 y publicado 28 de enero 2022

\section{RESUMEN}

El estudio tiene como objetivo analizar el aporte de la pragmática y el sentido del contexto en la comprensión de textos con la finalidad de caracterizar la naturaleza del texto considerando dos ideas: la comunicación no convencional y la naturaleza del texto como insumo para PISA. Se ha utilizado el método narrativo-biográfico en tanto los resultados desfavorables caracterizan las limitaciones de escolares peruanos al encarar la prueba PISA 2018. Se ha valorado la narrativa existente de los resultados a través del análisis documental en cuanto a los aportes de la pragmática y su funcionalidad. Los resultados cualitativos reflejan que la prueba PISA 2018 no considera el contexto como insumo para seleccionar los textos para evaluar la comprensión. A modo de conclusión, los resultados obtenidos en la prueba PISA 2018 no reflejan el nivel de comprensión de los escolares.

Palabras clave: Pragmática; Contexto; Comprensión de textos

\section{ABSTRACT}

The study aims to analyze the contribution of pragmatics and the sense of context in text comprehension, with the purpose of characterizing the nature of text considering two ideas: unconventional communication and the nature of text as an input for PISA. The narrativebiographical method has been used as unfavorable results characterize the limitations of Peruvian schoolchildren when facing the PISA 2018 test. The existing narrative of the results has been assessed through documentary analysis in terms of the contributions of pragmatics and its functionality. The qualitative results reflect that the PISA 2018 test does not consider context as an input for selecting texts to assess comprehension. As a conclusion, the results obtained in the PISA 2018 test do not reflect the comprehension level of schoolchildren.

Key words: Pragmatics; Context; Text comprehension

\section{RESUMO}

O estudo visa analisar o contributo da pragmática e o sentido de contexto na compreensão dos textos, de forma a caraterizar a natureza do texto considerando duas ideias: a comunicação não convencional e a natureza do texto como insumo para PISA. Utilizou-se o método narrativo-biográfico porque os resultados desfavoráveis caracterizam as limitações das crianças peruanas em idade escolar quando enfrentam o teste PISA 2018. Valorou-se a narrativa existente dos resultados através da análise documental em termos dos contributos da pragmática e da sua funcionalidade. Os resultados qualitativos refletem que o teste PISA 2018 não considera o contexto como insumo para selecionar os textos para avaliar a compreensão. Em conclusão, os resultados obtidos no teste PISA 2018 não refletem o nível de compreensão dos escolares.

Palavras-chave: Pragmática; Contexto; Compreensão de texto 


\section{INTRODUCCIÓN}

El desarrollo de la comprensión de textos demanda la aprehensión de los procesos cognitivos $\mathrm{y}$ didácticos de parte del docente; sin embargo, el aporte de la pragmática no se considera como elemento significativo que permita comprender que el contexto de cada escuela rural o urbana se deba considerar. El problema de la no comprensión de textos ha permitido describir que los escolares tienen bajo rendimiento o simplemente se los califica de acuerdo con los resultados; no obstante, se ha dejado de lado la pragmática como una categoría epistemológica asociada a la competencia "comprende" y los mismos cambios que ha sufrido con el tiempo, porque se trata de una categoría muy amplia y compleja para evaluar.

Seguidamente, la competencia, se entiende como la "comprensión, el uso y la reflexión a partir de los textos escritos [...] posteriormente, se añade el compromiso [...], finalmente, incorporan la evaluación" (MINEDU, 2018, pp. 15-16). Este proceso evolutivo conceptual deja en claro que la comprensión no es una competencia estática y requiere que otras ciencias como la pragmática aporten al sentido y mecanismo de comprensión.

Sin embargo, se resalta que las tres categorías (comprensión, compromiso y evaluación) evidencian la neutralidad de los textos escritos, pero al mismo tiempo define la ambigüedad de la categoría que, con el tiempo, ha cambiado progresivamente; no obstante, "es necesario recurrir a información pragmática para recrear mentalmente una situación comunicativa en la que éstos cobren sentido" (Murray et al., 2015, p. 52), porque no es suficiente el aporte teórico cuando los resultados son siempre desfavorables. La utopía del concepto de competencia no permite decodificar los resultados de la incorrecta comprensión de los textos que plantea PISA. Las tres categorías caracterizan la comprensión de textos como objeto material de la pragmática y de la misma hermenéutica. Para ello, el estudio se basa en dos ideas centrales: a. la comunicación no convencional y b. la naturaleza del texto. Estas dos ideas son tratadas desde la perspectiva de la importancia de la pragmática en el entendido que "la importancia del lenguaje radica en las implicaciones de las actitudes, las que se basan en los modelos o tipos de crianza y de educación en diferentes esferas"(Calua, Delgado y López, 2021, p. 317), de tal manera que pretender que los estudiantes comprendan lo que leen no depende, estrictamente, de lo que haga el docente o deje de hacer, sino que las condiciones de la pragmática se ha soslayado discretamente.

Bajo esta perspectiva, el estudio aborda como problema el siguiente interrogante: ¿cuáles son las limitaciones en la comprensión de textos al no considerar el aporte de la pragmática? El cuestionamiento está focalizado dentro de las prácticas evaluativas de PISA y las limitaciones propias de las prácticas docentes en el sistema educativo peruano.

La inquietud planteada pretende esclarecer y situar el aporte de la pragmática dentro del proceso de construcción de preguntas y la resolución de las mismas considerando que el contexto es un factor definitivo. En este sentido, se plantea como objetivo: analizar el aporte de la pragmática y el sentido del contexto en la comprensión de textos, considerando que los resultados son desfavorables, considerando que, en lo sucesivo, se afecta la objetividad de la evaluación. El aporte de la pragmática al proceso 
de construcción de preguntas cuyo objetivo es evaluación el nivel de comprensión debe ser considerado como categoría funcional porque es parte de la construcción social y cultural de los escolares.

\section{METODOLOGÍA}

El método empleado es el narrativobibliográfico considerando que los resultados PISA 2018 constituye el testimonio de actuación de miles de escolares de 79 países, en el que se encuentra en Perú con una puntuación inferior al establecido por PISA. La estructura narrativa de los documentos analizados (informe PISA 2018 y los referentes teóricos sobre la pragmática) no corresponden a documentos de acceso cerrado, por el contrario, son de dominio público y por tanto permite la investigación con enfoque cualitativo. La información utilizada tampoco es de acceso restringido por cuanto se puede obtener libremente de la Internet. El informe PISA 2018 expuesto a la luz del conocimiento y perspectiva de la investigación se corresponden con la dimensión heurística porque se ha conocido que las dos categorías: la comunicación no convencional y la naturaleza del texto son necesarias para poder evaluar el nivel de comprensión, razón por la cual la metodología se sustenta en dos procesos: el razonamiento inductivo y el deductivo. El primero refiere a las prácticas evaluativas de PISA a nivel mundial con mismo formato, en cuanto al razonamiento deductivo es el que conduce reflexionar sobre el aporte e importancia de la pragmática en la construcción de preguntas con fines de evaluación del proceso de comprensión.
El corpus del estudio tiene como referencias el aporte de la pragmática al proceso de elaboración de preguntas por parte de PISA, considerando dos elementos: a) la comunicación no convencional y b) el texto y su naturaleza. En cuanto a la muestra se precisa que se trata de 342 instituciones educativas peruanas, de las cuales el $70 \%$ con instituciones estatales y $30 \%$ son de régimen privada. Se ha tomado como muestra a 8028 estudiantes con el fin de valorar el nivel de comprensión. La muestra ha tomada del informe PISA 2018, puesto que se trata de los resultados oficiales.

\section{DESARROLLO Y DISCUSIÓN}

\section{La comunicación no convencional}

Los procesos comunicativos dentro del proceso formativo tienen particularidades centradas en la formalidad del lenguaje que provocan comentarios adversos cuando los resultados no demuestran que la sociedad haya desarrollado todas sus competencias, durante el proceso formativo, los escolares transitan 14 años dentro del proceso que demanda el sistema educativo peruano, tiempo suficiente para comprender que sí es posible que el nivel de comprensión sea satisfactorio; sin embargo, los problemas son recurrentes, porque en Perú no manifiesta el desarrollo de competencias en referencia a la comprensión de lectura. Este proceso tiene elementos clave y que están bien definidos con el mismo propósito de uniformizar las ideas en cuanto a comprensión. PISA sostiene que se trata de la "capacidad para comprender, utilizar, reflexionar e interesarse por los textos escritos, para alcanzar los propios objetivos, desarrollar el conocimiento 
y potencial personales, y participar en la sociedad". Esta percepción se ha logrado del estudio realizado en 79 países. En el caso de América Latina, Perú se ubica después de Chile, Uruguay, Costa Rica, México, Brasil, Colombia y Argentina.

ElhallazgonoesfavorableporquePerúnoalcanza al nivel mínimo de 407 puntos, solo ha logrado 401 puntos en comprensión de lectura. La controversia tiene muchas explicaciones que son recurrentes en el contenido o en los mismos conceptos que están alrededor del proceso de comprensión o en todo caso cerca de la comunicación como actuación humana que trasciende como proceso operacional, puesto que, el acto de comprender es la aplicación de muchas ciencias conexas que bien pueden explicar los resultados. En este sentido, es pertinente juzgar el rol del maestro mediante el siguiente interrogante ¿en qué medida la valoración de la pragmática contribuye a mejorar las competencias en los docentes para mejorar el proceso de comprensión de textos?

Se entiende que "la comunicación no se realiza mediante oraciones ni sumas de oraciones y hay fenómenos lingüísticos determinantes en el intercambio comunicativo que no se pueden tratar desde la sintaxis" (Yanguas, 2009, p. 3), porque las actuaciones humanas no solo se restringen al poder de la gramática. El proceso comunicativo no responde a la pureza de la gramática, tampoco se trata de valorar que la comunicación existe si las oraciones son propias de los propósitos comunicativos. El riesgo de la comprensión de textos radica en el hecho de comprender que se trata de un proceso humano $y$, por tanto, es el objeto de estudio para las ciencias sociales; sin embargo, los ejercicios pragmáticos no son considerados para el análisis de los procesos cognitivos, dado que estos existen dentro de contextos en los cuales el hombre se desarrolla. Por ejemplo, la posibilidad o imposibilidad de comunicarse solo con señas, donde la sintaxis no es funcional; sin embargo, se afirma que el proceso comunicativo es un producto socio-cultural.

La escuela es el espacio secular que desarrolla formas y mecanismos para superar las necesidades, esta condición solo existe siempre y cuando los modos de actuar del docente y estudiantes son pertinentes, desde luego que las "estrategias como el diálogo constante, la pedagogía de la pregunta y ejercicios de lectura y escritura, las cuales deben ser fortalecidas para permitir una mayor apropiación de la función comunicativa..." (Palacio, 2018, p. 146) son ajenas a la constitución del texto, porque cada idea que trasciende en las decisiones de las personas es notoria mediante la naturaleza del texto sin importar el concepto. El proceso de comprensión, al parecer, no se relaciona con la pedagogía de la pregunta, en tanto que el planteamiento de PISA radica en condiciones propias (contenidos, procesos, contextos) del texto. Al valorar los resultados de 342 instituciones educativas peruanas $(70 \%$ estatales y $30 \%$ privadas), las mismas que reunieron a 8028 estudiantes no representa el ejercicio del docente en el aula mediante el poder de la pedagogía, lo cual desnaturaliza el rol cuando intenta mejorar el proceso de comprensión.

La utilidad de la pragmática no es evidente porque el docente desconoce el valor que tiene sobre los procesos cognitivos, los mismos que definen el estatus de la comprensión. Las relaciones entre comprensión y pragmática no están contempladas porque el proceso de comunicación 
es ajeno al proceso de comprensión; es decir, el acto comunicativo marcha independientemente del proceso de comprensión; sin embargo, las referencias del sentido de la pragmática afirman que el lenguaje, dentro de la comunicación, no es una categoría sencilla; por el contrario, es regulado en el sentido que al utilizar el lenguaje surgen condiciones "que determinan tanto el empleo de un enunciado concreto por parte de un hablante concreto en una situación comunicativa concreta, como su interpretación por parte del destinatario" (Escandell, 1996, citado en Murillo, 2004, p. 3). Es necesario entonces cuestionarse cuando se habla de comunicación concreta, ¿no es acaso el proceso por el cual el hombre se comunica suficientemente? Bajo esta premisa, ¿es posible cuestionar los resultados de PISA al utilizar contenidos, procesos y contextos?, ¿estas tres categorías permiten comprender la existencia de una comunicación concreta, frente al hablante concreto?, ¿un hablante, al momento de comunicarse, realiza una acción concreta? No es sencillo asumir que un estudiante peruano no demuestre sus competencias porque "el resultado de ignorar las diferencias en los significados intersubjetivos puede ser desastroso [porque interpretar] a otras sociedades con las mismas categorías que interpretamos la nuestra" (Taylor citado en Packer, 2018, pp. 181-182), nada más razonable que el propio cuestionamiento al enfoque de la comunicación mediante una prueba que busca evaluar el nivel de comprensión de información.

El nivel de concreción u objetividad del hombre y la comunicación implica aprehender que, desde siempre "la competencia comunicativa es la habilidad para utilizar la lengua en la interacción social, basada en la negociación de significados entre dos hablantes" (Berenguer, Roca, Torres, 2016, pp. 29-30) que pretenden entender la realidad. Las habilidades que exige PISA son ajenas al contexto real donde la negociación social entre los hablantes no es la mejor porque los resultados no son favorables; no obstante, se trata de una competencia que, por obvias razones se deduce que comprender representa el concepto más absoluto del razonamiento humano; aunque muchos demuestren limitaciones para ello porque, en realidad, se trata de representaciones como el mismo lenguaje.

Por otro lado, "Los modelos del contexto controlan la producción y la recepción del discurso, de tal manera que la estructura del discurso (o su interpretación por el receptor) sea 'adecuada' o 'apropiada' a la situación interpersonal y social” (Van Dijk, 2001, p. 73); por lo tanto, la importancia de la pragmática en la construcción de la comunicación y en el ámbito de la escuela es ajena y utópica al intentar valorar el nivel de comprensión de textos; puesto que, un texto es el reflejo humano de su propia evolución. Esta construcción opuesta al capital cognitivo del individuo define que la construcción social no se restringe solo a la pregunta y respuesta que PISA pretende, es todo lo contrario porque el hombre se refleja por medio del texto y este construye la identidad del individuo mediante la representación desarrollada por el lenguaje, puesto que se trata de un conjunto de símbolos, muchas veces antitéticos y controversiales. Por ejemplo, cuando se intenta comprender el sentido de la metáfora en la letra de las canciones; sin embargo, trasciende en el mensaje cuando "dicen que amar quita el hambre" (ver traducción en: https://www. youtube.com/watch?v=RRs6ju42Icg) o la siguiente 
carta que fue motivo de una pregunta planteada por PISA.

Estoy Indignada porque es la cuarta vez que han tenido que limpiar y volver a pintar la pared del colegio para quitar las pintadas. La creatividad es admirable, pero la gente debería encontrar otras formas de expresarse que no suponga gastos extra para la sociedad. ¿Poe qué nos creáis esa mala reputación a los jóvenes pintando en lugares en los que está prohibido? Los artistas profesionales no cuelgan sus cuadros en las calles, ¿verdad? Lo que hacen es buscar quien les financie y hacerse famosos a través de exposiciones que sí son legales. En mi opinión, edificios, vallas y bancos de los parques son obras de arte en sí mismos. Es realmente patético estropear su arquitectura con graffiti y lo que es más, el método que se utiliza para ello destruye la capa de ozono. Realmente no puedo entender por qué estos artistas delincuentes se enfadan cuando les quitan sus "obras de arte" de las paredes una y otra vez.

\section{Pregunta 1}

Olga.

El propósito de estas cartas es...

A Explicar lo que son las pintadas o graffiti.

B Presentar una opinión sobre las pintadas o graffiti.

C Demostrar la popularidad de las pintadas o graffiti.

D Decirle a la gente cuánto cuesta borrar las pintadas o graffiti.

Ambos ejercicios se definen como representaciones propias del lenguaje que busca que la comprensión sea uniforme y con ello las respuestas sean satisfactorias. La pragmática del texto genera especulaciones de las cuales se desprenden percepciones, que a su vez están asociadas con los saberes previos y con ello se genera una posible respuesta, porque el contexto es determinante.

Esto es pragmatismo del texto porque representa la humanidad indistintamente del lugar donde se haya desarrollado, la escuela tiene el mismo potencial y limitaciones que ofrecer con el fin de mejorar todos los procesos cuando consideren que la comprensión sin ella no es funcional. El proceso de comprensión tiene implicancias complejas porque son productos humanos donde "la relación entre comunicación y lenguaje es bidireccional y que se trata de una relación de interdependencia" (Monfort, 2009, p. 55), donde el ejercicio de poder es constante cuando existen estudiantes que no comprenden cuestionamientos o ideas con interpolaciones y códigos subyacentes al conocimiento científico o el lenguaje doméstico, la interdependencia es natural en tanto la condición de sujeto subordinado a las limitaciones de la escuela no busque los cambios inmediatos, esto es observar que la pragmática cumpla el papel sin ser ciencia porque no necesita serlo para comprender que la naturaleza humana es diversa; "aun cuando los seres humanos tenemos la capacidad de comunicar, este proceso no es adoptado con frecuencia o las formas en las que se realizan no son del todo las adecuadas y el mensaje que se desea transmitir no es captado en su totalidad" (Alba, Fajardo, Tixtha y Papaqui, 2012, p. 139) porque el conocimiento es amplio y no deja de ser complejo, la controversia entre lo útil para el desarrollo humano siempre se puede cuestionar porque existen formas absolutas de desarrollar el conocimiento y formas relativas para comprenderlos. 
Saber desarrollar el proceso de comprensión mediante un conjunto de estrategias pertinentes implica suficientemente que tanto el docente como los estudiantes busquen las mejores condiciones para desarrollar competencias con el fin de configurar el sentido de la competencia comunicativa porque, en realidad requiere "de una visión holística alejada de posturas mecanicistas y rígidas para dar paso a una multifactorial y necesariamente subjetiva o por lo menos flexible" (Bermúdez y Gonzáles, 2011, p. 99) con el fin de comprender el complejo mundo de las preguntas que buscan medir la comprensión estandarizada.

\section{El texto, su naturaleza}

Las condiciones polifónicas del texto establecen ciertas características que se deben tener cuenta con la finalidad de situar el uso pragmático de los conceptos. Comprender un texto no necesariamente es valorar la linealidad de las ideas, porque se trata de muchas con diversos enfoques del creador. Cada autor hasta puede ser arbitrario para plantear las ideas con el objeto de ser comprendidas; sin embargo, existe la necesidad de comprender que "[1]a pragmática, cuya característica esencial es considerar el lenguaje en su dimensión práctica, de uso..." (Shriffin 1994, citado en Murillo, 2004, p. 3) con el fin de ser precisos en la comprensión, aunque las valoraciones estén direccionadas solo al nivel literal de la comprensión. La naturaleza del texto es multidimensional porque se trata de una construcción socio-cultural y al mismo tiempo es el producto del desarrollo humano. Por ejemplo, determinar cuánto o qué se comprende, críticamente, un texto argumentativo no es lo mismo comprender el sentido de las ideas en un texto expositivo. Comprender éste último no implica necesariamente identificar los argumentos porque la exposición es solo eso: ex-poner, algo así como sacar a la luz lo que no es evidente.

El aporte de Shirffin es esclarecedor y el pragmatismo es evidente en tanto que existen "aquellos enfoques estructuralistas en los que se considera a la lengua como una abstracción, un sistema que puede ser solo definido a partir de las relaciones que sostienen unos elementos con otros" (Shriffin 1994, citado en Murillo, 2004, p. 3). Esta antípoda genera, en la percepción humana, una contradicción considerando el valor de la semántica de todas las ideas o de la propiedad del mensaje que se desea comprender porque, en cualquier texto, la lengua siempre será una abstracción arbitraria y contradictoria a las expectativas para optimizar la comprensión.

Todos los procesos cognitivos demandan otras condiciones vinculadas con el desarrollo mental, por ello es que la identificación de las ideas principales (IP) implica comparar con las ideas secundarias (IS), que a su vez se comparan con las ideas terciarias (IT); sin embargo, aun cuando se pueda discriminar, según su importancia, tienen el mismo valor dentro del todo representado en el texto. La comprensión, entonces es un proceso cognitivo que se basa en la comparación, la misma que "constituye una representación mental, o subtipo de exposición de información, que sugiere la búsqueda de elementos que se oponen, en su estructura, en su funcionamiento, en su procedencia o en su utilidad" (Álvarez y Ramírez, 2010, p. 75). Al mismo tiempo, el lector compara con sus propias ideas que devienen de la empírica, del error o cualquier otra percepción. Por ejemplo, 
"la comparación y el método comparativo no se utilizan solo en las ciencias naturales y físicas, sino que, a partir del positivismo y del nacimiento de las ciencias sociales se convierten en elementos cruciales de las nuevas ciencias sociales" (Tosolini, 2014, p. 41), ¿cómo es que se convirtieron en elementos cruciales?, este cuestionamiento permite valorar que la comprensión de textos se sustenta en la comparación de ideas que, muchas veces, no siempre se comprenden y se fuerzan a lograrlo porque la necesidad de comprender es cada vez más compleja sin considerar que la cognición humana requiere de mucho entrenamiento.

La madurez epistemológica conduciría al docente a lograr un perfil que permita comprender que el lenguaje no solo trasciende por el tipo de mensaje porque debe entenderse que "[1]a base texto es una representación del significado del texto centrada en el texto mismo" (Molinari y Duarte, 2007, p. 166); es decir, es una entidad propia de su creador y representa la filosofía dentro de un tiempo y espacio determinados. Por ejemplo, el análisis del mensaje de El Principito implica identificar cuál sería el mensaje, que no es el mismo que el docente haya identificado como producto de sus comparaciones consigo mismo o que haya realizado comparaciones con otros títulos. En realidad, "lo que determina el tipo de texto es la intención, la finalidad última para lo que ha sido construido; los fenómenos lingüísticos y textuales que materializan o muestran la intención del texto" (Álvarez, Perelló, y Pintos, 2007, pp. 45-46); por lo tanto, cuando se busca que los estudiantes comprendan la realidad del texto solo se pretende movilizar la cognición al amparo de la libertad y que ello construya la identidad sin someterse a la arbitrariedad de la representación lingüística porque todo texto creado es una respuesta a las necesidades individuales y no necesariamente se corresponde con las del estudiante.

Analizar las relaciones como producto de las comparaciones implica tener en cuenta que el proceso de comunicación y las condiciones del texto son elementos valorados por la pragmática con el fin de reconocer que la comprensión de textos es un hecho socio-cultural porque "la variedad de textos que pueden existir derivan [deriva] de la realización del discurso y el texto como un proceso de interacción y como un producto retóricogramatical de la situación comunicativa" (Carrillo, 2016, p. 14), ello es una oportunidad para que la comprensión otorgue la misma posibilidad porque se trata de la interacción humana que se produce en cualquier escenario dado que "el verdadero enriquecimiento lingüístico-comunicativo vendrá no por el análisis de determinados textos sino por las redes y asociaciones que establecemos entre ellos, yendo más allá de la mera forma textual" (Rico, 2005, p. 86), lo que quiere decir que la comprensión sin intención no tiene razón de ser si es que procura que los escolares desarrollen competencias. Desde la pragmática, entonces, es necesario identificar todos los procesos cognitivos movilizados para determinar el rol del pensamiento y que ello determine otros resultados con el fin de formar la persona. Dentro de las prácticas orientadas a mejorar la comprensión debe estar el reconocimiento conceptual del texto, con el fin de que los estudiantes no desarrollen aversión a la comprensión y por tanto la escuela tendría la misma valoración. Debería entender que "la comunicación, además de darnos la oportunidad de codificar y descodificar, nos debe conducir 
a expresar y reconocer intenciones, a realizar procesos inferenciales que nos permitan ir más allá de lo codificado" (Fajardo, 2009, p. 129) con la finalidad de desarrollar el pensamiento crítico, ello no es posible con las condiciones establecidas por la misma gramática, porque se trata de valorar que la comunicación es una necesidad humana no solo con el fin de estar conectados, se trata de una forma de vida donde los códigos deben estar a disposición del mismo aprendizaje y desarrollo humano.

De este recorrido orientado a valorar el proceso de comprensión, no se debe descartar que "las teorías de la Pragmática, por tanto, pretenden estudiar ese comportamiento caótico sobre la base del descubrimiento de leyes de cumplimiento regular (y general)" (Zamorano, 2012, p. 691) con el fin de mejorar la percepción humana acerca del amplio significado de la comprensión de textos y que no sea el tránsito o recorrido lineal de las ideas ya sea vertical $u$ horizontal para descubrir la idea que pretende el autor porque un texto no solo es la representación particular del pensamiento del creador, se trata de una entidad lingüística prolija que trasciende no tanto por quien lo escribió, sino por el valor del impacto al momento de comprender las ideas.

Buscar respuestas ideas al margen del contexto es impertinente porque "cada intérprete necesita introducirse en la dimensión social y la dimensión individual del autor para comprenderlo". (Martínez, 2004 citado en Alarcón, 2015, p. 3), caso contrario las respuestas no serían suficientes para las expectativas del evaluador, las consecuencias no serían nada agradables. Se trata de un proceso con arraigo de la pedagogía y de la misma didáctica, el contexto nunca ha sido ajeno a los propósitos formativos. Sin embargo, "En la medida en que el lector se identifique con el autor y se ponga en su lugar, tanto mejor será la interpretación" (Martínez, citado en Alarcón, 2015, p. 3), es evidente que la construcción dialéctica de la pregunta que busca una respuesta taxativa es hasta surrealista porque privilegia el poder del maestro frente a la desventaja del estudiante al no comprender que la pragmática es necesaria.

\section{Discusión}

La valoración de los procesos cognitivos que demanda la comprensión de textos desconociendo la importancia del contexto conduce a la incertidumbre toda vez que comprender información requiere de ciertas habilidades y conocimientos, se trata de dos condiciones preestablecidas que se debe considerar. Por ello es definitivo que el contexto en el cual se desarrolla la escuela, de modo general, sea considerado al momento del uso del lenguaje y lo significativo que es valorar la representación del pensamiento mediante el lenguaje. Cuando se pregunta para buscar resultados de cómo o qué es lo que se comprende se debe considerar que el lenguaje es la construcción sociocultural; por lo tanto, nadie tiene el valor de verdad sobre los significados. El lenguaje es mucho más que la transmisión de ideas con fines de comprobación, se trata de entender que el contexto no tiene límite porque es cualquier circunstancia influyente directa o indirectamente en el consciente humano. Interpretar una pregunta no es lo mismo que interpretar el pensamiento del creador de la misma, porque se trata de una representación humana que tiene pretensiones formales para valorar le evolución de la cognición mediante una pregunta. 
Todo proceso de comprensión tiene sus propias circunstancias, de hecho, las "dificultades en los procesos básicos atencionales y perceptivos pueden condicionar, desde el principio del desarrollo, el aprendizaje pragmático-comunicativo" (Casas, Fernández y Remírez, 2004, p. 111); sin embargo, los juicios del docente condenan los resultados cuando los estudiantes no responden a las expectativas. Preguntar y responder es un juego arbitrario, donde la hegemonía del docente no tiene el control de sus expectativas que se fundan en la misma construcción dialéctica. Se debe precisar que "la mayoría de los infortunios o malentendidos entre los hablantes repercuten en la comprensión y entendimiento, que muchas veces ocasionan fracasos conversacionales e incluso rompimiento de la comunicación" (Moya, 2009 , p. 243). Las consecuencias siempre terminan etiquetando a los estudiantes como aquellas personas con serias dificultades. El infortunio, finalmente, recae en la caracterización negativa del proceso de comprensión. La discusión sobre el controversial contexto se debe tener en cuenta con la finalidad de valorar el papel de la pragmática cuando se construyen preguntas que pretenden caracterizar el proceso cognitivo de los estudiantes.

\section{CONCLUSIONES}

El proceso de evaluación de la comprensión de información desarrollado por PISA necesita incorporar el aporte de la pragmática, toda vez que un texto, cual sea su estructura es una representación mental que guarda elementos propios del razonamiento del creador. Por lo tanto, comprender exactamente o lo más próximo a ello es arbitrario, porque el texto, tanto en la forma y contenido, ofrece apertura y libertad para entender la intención del interlocutor, antes que la rigurosidad del contenido, toda vez que el contexto es un factor determinante en la comprensión del lenguaje como representación relativa del conocimiento. Por otro lado, la pragmática es parte de la polifonía del texto porque el creador recrea las ideas dentro de su propio contexto, de tal manera que un texto como medio para evaluar el proceso de comprensión no es suficiente si es que no se considera el antes o lo que trasciende en la cognición del creador. Del mismo modo, el texto es, en sí mismo, la representación etnográfica que solo define cierta caracterización del autor y que para comprenderlo absolutamente puede ser un peligro para el desarrollo del conocimiento en edad escolar. Finalmente, el estudio deja abierta la posibilidad hacia nuevas aristas de investigación sobre la caracterización del contexto como elemento insoslayable y concomitante al proceso de comprensión.

\section{REFERENCIAS}

Alarcón Puentes, Johnny (2015). La hermenéutica como anclaje epistémico del método etnográfico. Vivat Academia, (133),1-11. [fecha de Consulta 28 de Julio de 2021]. ISSN: Disponible en: https:// www.redalyc.org/articulo.oa?id=525752885001

Alba, A., Fajardo, G., Tixtha, E. y Papaqui, J. (2012). La comunicación enfermera-paciente en la atención hospitalaria, caso México. Enfermería Neurológica, 11(3), 138-141. https://revenferneurolenlinea.org.mx/index. php/enfermeria/article/view/144/144

Álvarez, T., Perelló, D. y Pintos, M. (2007). De cómo convertir un texto expositivo en argumentativo y sus implicaciones didácticas. Didáctica (lengua y Literatura), 19, 31-46. http://www.ehu.eus/ PAT/compe/lanak/DIDA0707110031A.pdf

Álvarez, T. y Ramírez, R, (2010). El texto expositivo y su escritura. Revista Folios, (32), 73-88. https:// www.redalyc.org/pdf/3459/345932035005.pdf 
Berenguer, I., Roca, M. y Torres, I. (2016). La competencia comunicativa en la enseñanza de idiomas. Dominio de las Ciencias, 2(2), 25-31. https://dialnet.unirioja.es/descarga/ articulo/5761589.pdf

Bermúdez, L. y González, L. (2011). La competencia comunicativa: elemento clave en las organizaciones. Quórum académico, 8(1), 95-110. https://www.redalyc.org/ pdf/1990/199018964006.pdf

Calua, M., Delgado, Y., López, Ó. (2021). Comunicación asertiva en el contexto educativo: revisión sistemática. Redipe, 10(4), 315-334. https://doi.org/10.36260/rbr.v10i4.1274

Casas, M., Fernández, Y., Remírez, M. (2004). Complejidad gramatical y mecanismos de cohesión en la pragmática comunicativa de los niños con trastorno por déficit de atención con hiperactividad. Rev Neurol, (38), 111-116 https://n9.cl/irasp

Carrillo Guerrero, L. (2016). Actualización retórica de la lengua: el registro. TonosDigital,9, 1-21. https://digitum.um.es/digitum/ bitstream/10201/49914/1/Actualizacion\%20 retorica.pdf

Fajardo, L. (2009). A propósito de la comunicación verbal. Forma y función, 22(2), 121-142. https:// dialnet.unirioja.es/descarga/articulo/6454284. pdf

MINEDU (2018). Evaluación Pisa 2018. Recuperado de: http://umc.minedu.gob.pe/ wp-content/uploads/2019/12/Resultados-PISA2018-Per\%C3\%BA.pdf

Molinari, C. y Duarte, A. (2007). Comprensión del texto narrativo e inferencias. Subjetividad $y$ procesos cognitivos, (10), 163-183. https:// dialnet.unirioja.es/descarga/articulo/3147083. pdf

Monfort, I. (2009). Comunicación y lenguaje: bidireccionalidad en la intervención en niños con trastorno de espectro autista. Revista de neurología, 48(2), 53-56. https://faros.hsjdbcn. org/adjuntos/293.1-comunicacion_lenguaje.pdf

Moya, C. (2009). Aproximación pragmática a los conceptos de" acto de habla" y de" acción comunicativa". Revista de Psicoanálisis, (9),
229-243. https://dialnet.unirioja.es/descarga/ articulo/3808802.pdf

Murray, C., Tobar, A., Villablanca, F. y Soto, G. (2015). El componente pragmático en adultos con síndrome de Asperger: actos de habla indirectos, metáforas y coerción aspectual. Revista de lingüística teórica y aplicada, 53(1), 35-58. https://scielo.conicyt.cl/pdf/rla/v53n1/ art_03.pdf

Murillo Medrano, Jorge (2004). La pragmática y la enseñanza del español como segunda lengua. Revista Educación, 28(2),255-267. [fecha de Consulta 17 de Julio de 2021]. ISSN: 03797082. Disponible en: https://www.redalyc.org/ articulo.oa?id $=44028214$

Packer, M. (2018). La ciencia de la investigación cualitativa. DGP. Colombia

Palacio, A., Palacio, B., Perea, L. y Moreno, M. (2018). El componente pragmático: elemento indispensable para la lectura en contexto. Ciencias Sociales y Educación, 7(14), 127-147. https://doi.org/10.22395/csye.v7n14a7

Rico, A. (2005). De la competencia intercultural en la adquisición de una segunda lengua o lengua extranjera: conceptos, metodología y revisión de métodos. Porta Linguarum, 79-94. https:// digibug.ugr.es/bitstream/handle/10481/29122/ Rico_SegundaLengua.pdf?sequenc

Tosolini, A. (2014). Comparar una nueva lectura de la realidad plural. Narcea. Madrid.

Van Dijk, T. (2001). Algunos principios de una teoría del contexto. Revista latinoamericana de estudios del discurso, 1(1), 69-82. https://n9.cl/ zjzt6

Yanguas, L. (2009). El texto descriptivo en el aula de ELE. De la teoría a su presencia en el MCER y el Plan Curricular del IC. Marco ELE. Revista de Didáctica Español Lengua Extranjera, (8), 1-26. https://www.redalyc.org/pdf/921/92152527013. pdf

Zamorano, A. (2012). Teorías del caos y lingüística: aproximación caológica a la comunicación verbal humana. Signa (21), 679-705. https:// dialnet.unirioja.es/descarga/articulo/3831866. pdf 